\title{
A propos des conditions d'échantillonnage pour le dosage du delta-9-tétrahydrocannabinol dans les variétés de chanvre à usage industriel
}

\section{Comments on the sampling conditions for the titration of delta-9-tetrahydrocannabinol in varieties of hemp used for industrial purposes}

Gilbert FOURNIER*(1), Olivier BEHEREC ${ }^{(2)}$, Sylvestre BERTUCELLI ${ }^{(2)}$, Jean-Paul MATHIEU ${ }^{(2)}$

(1) Laboratoire de Pharmacognosie, UPRES 8076, CNRS (BioCIS), Faculté de Pharmacie, 5, rue J.-B. Clément - 92296 CHÂTENAY-MALABRY

(2) Fédération Nationale des Producteurs de Chanvre (FNPC), 20, rue P. Ligneul - 72000 LE MANS

* Auteur à qui adresser la correspondance : Professeur Gilbert FOURNIER, Laboratoire de Pharmacognosie, UPRES 8076, CNRS (BioCIS), Faculté de Pharmacie, 5, rue J.-B. Clément - 92296 CHÂTENAY-MALABRY Tél : 0146835597 - Fax : 0146835710 - E-mail : gilbert.fournier@cep.u-psud.fr

(Reçu le 22 octobre 2001 ; accepté le 24 novembre 2001)

\section{$R E ́ S U M E ́$}

L'originalité du chanvre (Cannabis sativa $L$.), tant d'un point de vue chimique que pharmacologique, est liée à la présence de substances spécifiques : les cannabinoïdes. Parmi la soixantaine de dérivés connus, le delta-9-tétrahydrocannabinol $(\Delta-9-T H C)$ est responsable des propriétés psychotropes de la plante. La teneur de cette substance est l'objet de variations importantes liées principalement à des facteurs génétiques (existence de différents types chimiques) mais aussi, dans une moindre mesure, à des facteurs écologiques. La détermination de la teneur en $\Delta-9-T H C$ était réalisée depuis 1990 selon un protocole officiel, les prélèvements étant effectués lors de la fin de la floraison, sur les tiers supérieurs de 500 plantes. Seules les cultures établies à partir de

\begin{abstract}
SUMMARY
The originality of hemp (Cannabis sativa L.), from a chemical and pharmacological point of view, is linked to the presence of specific substances : cannabinoids. Among the approximately sixty known compounds, only delta-9-tetrahydrocannabinol $(\Delta-9-T H C)$ is responsible for the psychotropic properties of the plant. The amount of this substance can vary due to both genetic (existance of differents chemical types) and, to a lesser degree, ecological factors.

Since 1990, $\Delta-9-$ THC has been harvested according to an official protocol: the samples had to be taken at the end of the flowering period taking the upper third of 500 plants. Only crops grown with varieties in which $\Delta-9-T H C$ level was not over $0.30 \%$ were accepted.
\end{abstract}


variétés dont la teneur en $\Delta-9-T H C$ n'était pas supérieure à $0,30 \%$ étaient autorisées.

En 2000, une nouvelle méthode officielle est entrée en vigueur. Deux méthodes différentes de prélèvements sont décrites. Une méthode A, pour les constatations au niveau de la production: prélèvement, entre les vingt jours après le début et les dix jours après la fin de la floraison, des 30 centimètres supérieurs de 50 plantes. Une méthode $B$, à suivre lors de l'inscription d'une nouvelle variété : prélèvement, au cours des dix jours suivant la fin de la floraison, des tiers supérieurs de 200 plantes. Ne sont désormais autorisées que les variétés dont la teneur en $\Delta-9-T H C$ n'est pas supérieure à $0,20 \%$.

Dans le chanvre, les teneurs en $\triangle-9-T H C$ étant très variables, la mise en cuvre des protocoles $A$ ou $B$ ne permet pas d'obtenir le même résultat lors du dosage du $\Delta-9-T H C$. Sur un plan strictement analytique, cela pose un réel problème. Par ailleurs les teneurs limites en $\triangle-9-T H C$ des variétés dont la culture est autorisée ont, dans le même temps, diminué, passant de 0,30 à 0,20\% réduisant ainsi d'autant la marge sur la répétabilité des analyses.

\section{MOTS-CLÉS}

Chanvre, Cannabis sativa, delta-9-tétrahydrocannabinol, dosage.

\section{Introduction}

Il est désormais classiquement admis que la principale substance responsable de l'activité psychotrope du chanvre (Cannabis sativa L.) est le $\Delta$-9-tétrahydrocannabinol $(\Delta-9-T H C)$. La détermination de la teneur en $\Delta-9$-THC permet donc d'évaluer le potentiel psychoactif d'un échantillon de chanvre.

Dans la plante, la teneur de cette substance est l'objet de nombreuses variations tant d'origine intrinsèque (d'un facteur 1 à un facteur qui peut être supérieur à 1000) qu'extrinsèque (d'un facteur 1 à un facteur 2 environ). De telles variations entraînent des difficultés lors de l'échantillonnage en vue de la mesure du taux de $\Delta$-9-THC. Parmi les variations intrinsèques, il convient de rappeler que chez le chanvre, selon les proportions relatives de $\Delta-9$-THC et de cannabidiol (CBD, cannabinoïde non-psychotrope, précurseur biogénétique du $\Delta-9-\mathrm{THC}$ ), on distingue plusieurs variétés chimiques (encore appelées types chimiques ou chimiotypes) (1)

Un arrêté du 22 août 1990 (paru au Journal officiel du 4 octobre 1990) précisait, dans un Article $1^{\text {er }}$ : «Sont autorisées au sens de l'article R. 5181 du code de la Santé publique, la culture, l'importation, l'exportation, l'utilisation industrielle et commerciale (fibres et graines) des variétés de Cannabis sativa $\mathrm{L}$. répondant aux critères suivants :
In 2000, a new official method come into force. Two different sampling methods are described. On the one hand, method A to establish the production level: samples have to be taken between twenty days after the beginning of flowering and ten days after the end of flowering, on the 30 uppermost cm of 50 plants. On the other hand, method $B$ is applied for the registration of a new variety : samples have to be taken during the 10 days following the end of flowering on the upper third of 200 plants. Only the varieties with a $\Delta-9-T H C$ level below $0.20 \%$ are accepted.

Because of the variability of $\triangle-9-T H C$ levels in hemp, the use of protocol $A$ or $B$ doesn't give the same result for $\triangle-9-T H C$ titration. From a strictly analytical point of view, this is a real problem. Furthermore in the mean time the maximal limit levels of $\triangle-9-T H C$ of authorized varieties have decreased from 0.30 to $0.20 \%$. The level on the repetability of the analyses have decreased accordingly.

\section{KEY-WORDS}

Hemp, Cannabis sativa, delta-9-tetrahydrocannabinol, titration.

$>$ le poids de THC (tétrahydrocannabinol) de ces variétés par rapport au poids d'un échantillon porté à poids constant n'est pas supérieur à 0,30 p.100;

$>$ la détermination du taux de tétrahydrocannabinol et la prise d'échantillons en vue de cette détermination sont effectuées selon la méthode unique prévue en annexe I».

L'article 2 mentionnait que «les variétés autorisées sont les suivantes : Carmagnola, C.S., Ferimon, DeltaLlosa, Delta-405, Fedora 19, Felina 34, Fedrina 74, Fibranova, Fibrimon 24, Fibrimon 56 et Futura».

De plus, en annexe, était décrite la «Méthode communautaire pour la détermination quantitative du $\Delta^{\prime}$ THC des variétés de chanvre» (dans la nouvelle nomenclature, le $\Delta^{\prime}$ THC est appelé $\Delta 9$ THC).

Cette méthode officielle prévoyait de prélever «dans une population d'une variété de chanvre donnée au moins $\underline{500 \text { plantes, }}$, de préférence en différents points et en excluant les bordures. Ces prélèvements sont effectués en pleine journée à la fin de la floraison». A partir de ce prélèvement, l'échantillon laboratoire est constitué, «en vue de la détermination du taux de $\Delta^{\prime} \mathrm{THC}$, par le tiers supérieur des plantes, ce tiers étant débarrassé des tiges et des graines» (2).

Un tel protocole tenait donc compte de l'influence que peut avoir la variabilité de la teneur en $\triangle-9$-THC sur la reproductibilité du résultat obtenu lors du dosage. Cette méthode précisait, notamment, le nombre de plantes à 
prélever, la partie de chaque plante à échantillonner et le moment où devait se faire la récolte. Par expérience, il est classiquement admis que ces trois facteurs sont ceux qui influencent le plus le résultat du dosage du $\triangle-9-T H C$ : un nombre important de plantes minimise l'influence chimiotypique. La fin de la floraison est un stade végétatif sous influence photopériodique, se produisant donc à période fixe, pour une variété donnée, dans un lieu de culture donné ; ce stade est donc facile à repérer. Dans les cultures industrielles, le tiers supérieur correspond à l'ensemble des parties vertes (celles qui renferment le $\Delta-9$-THC).

Cependant, cette méthode communautaire, qui prévoyait un dosage du $\Delta-9-T H C$ par chromatographie en phase gazeuse avec un équipement défini (colonne remplie), était devenue obsolète du fait de l'évolution de cette technique analytique. Il a donc été récemment entrepris l'élaboration d'une nouvelle méthode de dosage (avec une colonne capillaire) prévoyant, entre autres, de nouvelles conditions d'échantillonnage (3).

L'objectif de ce travail est de commenter ces nouvelles conditions d'échantillonnage figurant dans la méthode communautaire pour la détermination quantitative du $\Delta$-9-THC des variétés de chanvre, parue récemment au Journal officiel des Communautés européennes sous la forme d'une annexe au Règlement $C E \mathrm{n}^{\circ}$ 2860/2000 de la Commission du 27 décembre 2000.

\section{Rappel de quelques points principaux figurant dans la nouvelle méthode commu- nautaire pour la détermina- tion quantitative du $\Delta-9-\mathrm{THC}$}

\section{Objet et champ d'application}

La méthode sert à déterminer la teneur en $\Delta-9$-THC des variétés de chanvre. Selon le cas elle est appliquée selon une procédure $\mathrm{A}$ ou une procédure $\mathrm{B}$ décrites ci-après.

La méthode est basée sur la détermination quantitative par chromatographie en phase gazeuse du $\Delta-9-T H C$ après extraction par un solvant.

\section{Procédure $A$}

La procédure A est utilisée pour les constatations au niveau de la production.

\section{Procédure $B$}

La procédure B est utilisée lors de l'inscription dans la liste des variétés éligibles à l'aide.

\section{Échantillonnage}

\section{Prélèvements}

Procédure A : dans une population d'une variété de chanvre donnée, on prélèvera une partie de $30 \mathrm{~cm}$ contenant au moins une inflorescence femelle pour chaque plante sélectionnée. Le prélèvement s'effectue pendant la période comprise entre les vingt jours après le début et les dix jours après la fin de la floraison, pendant la journée, selon un parcours systématique permettant une collecte représentative de la parcelle, en excluant les bordures.

Procédure B : dans une population d'une variété de chanvre donnée, on prélèvera le tiers supérieur de chaque plante sélectionnée. Le prélèvement s'effectue au cours des dix jours suivant la fin de la floraison, pendant la journée, selon un parcours systématique permettant une collecte représentative de la parcelle, en excluant les bordures.

\section{Taille de l'échantillon}

Procédure A : pour chaque parcelle, l'échantillon est constitué par les prélèvements sur 50 plantes.

Procédure B : pour chaque parcelle, l'échantillon est constitué par les prélèvements sur 200 plantes.

De plus, le Règlement prévoit que la teneur en tétrahydrocannabinol des variétés admises ne peut être supérieure à $0,20 \%$ et mentionne que les variétés de chanvre destiné à la production de fibres sont : Carmagnola, Dioica 88, Epsilon 68, Fedora 17, Fedrina 74, Ferina 32, Ferimon, Fibranova, Fibrimon 24, Fibrimon 56, Futura, Futura 75, Santhica 23.

\section{Problématique de l'échan- tillonnage pour une mesure du $\Delta-9-$ THC}

Rappelons, d'une part, qu'un tel échantillonnage doit être représentatif de la variété et/ou du lot de semences et/ou de la culture et que, d'autre part, les cannabinoïdes, en particulier le $\Delta-9-T H C$, ne sont présents que dans les feuilles et les inflorescences. Il faut donc exclure de l'échantillon toutes les parties non vertes (tiges, graines, ...).

\section{Influence du nombre de plantes dans l'échantillon :}

La teneur en $\Delta-9$-THC d'un échantillon est, comme cela a été précédemment rappelé, considérablement influencée par la répartition chimiotypique au sein de la variété. 
La Figure 1 présente les résultats des dosages de $\Delta$-9-THC effectués sur des échantillons de taille variable. Les prélèvements ont été réalisés en 2000 , sur la variété Félina 34 , en prenant le $1 / 3$ supérieur des plantes (20 répétitions pour chaque prélèvement).

On observe sur la Figure 1 que, quel que soit le nombre de plantes prélevées, la moyenne des mesures est identique, mais que, plus le nombre de plantes dans l'échantillon est faible, plus la variation autour de la moyenne est importante, ce qui a pour conséquence une très mauvaise reproductibilité des résultats.

De même, la Figure 2 présente les résultats expérimentaux avec la variété Fédora 17 (variété sélectionnée depuis de nombreuses années sur des critères chimiotypiques, présentant une teneur en $\Delta-9$-THC constamment faible). Chez une telle variété, il ne doit donc y avoir aucun impact du nombre de plantes dans l'échantillon sur la valeur obtenue. On constate effectivement un tel résultat (moyenne et écart type constants et très faibles).

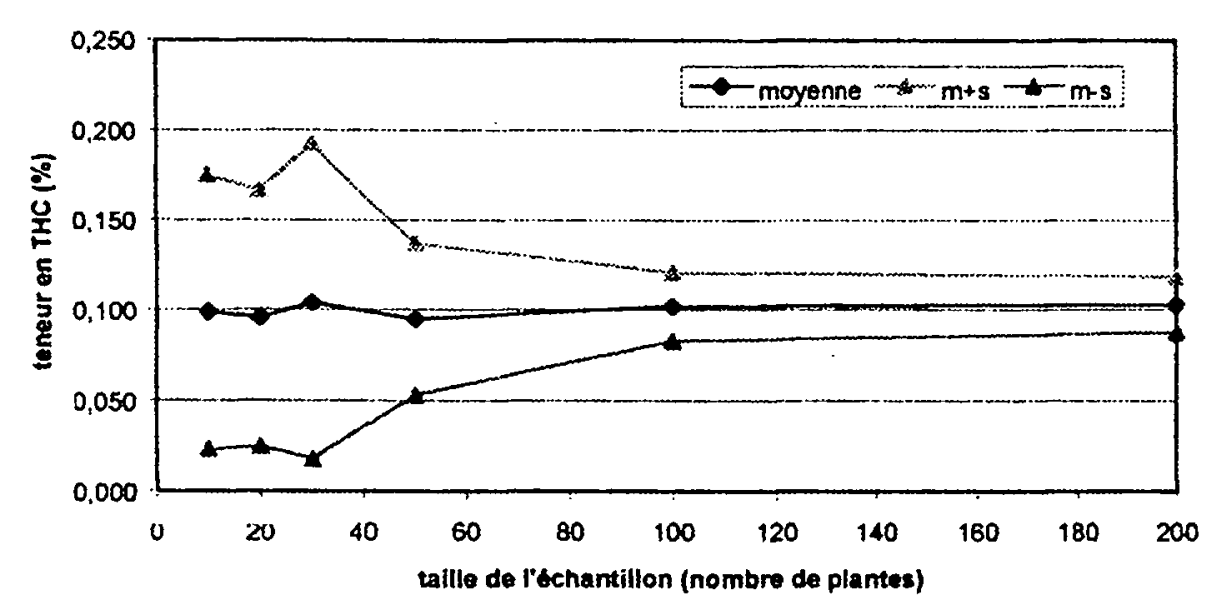

Figure 1 : Fluctuation de la teneur en $\Delta-9-T H C$ en fonction de la taille de l'échantillon. Variété Félina 34 - Prélèvement fin floraison des tiers supérieurs - Année 2000.

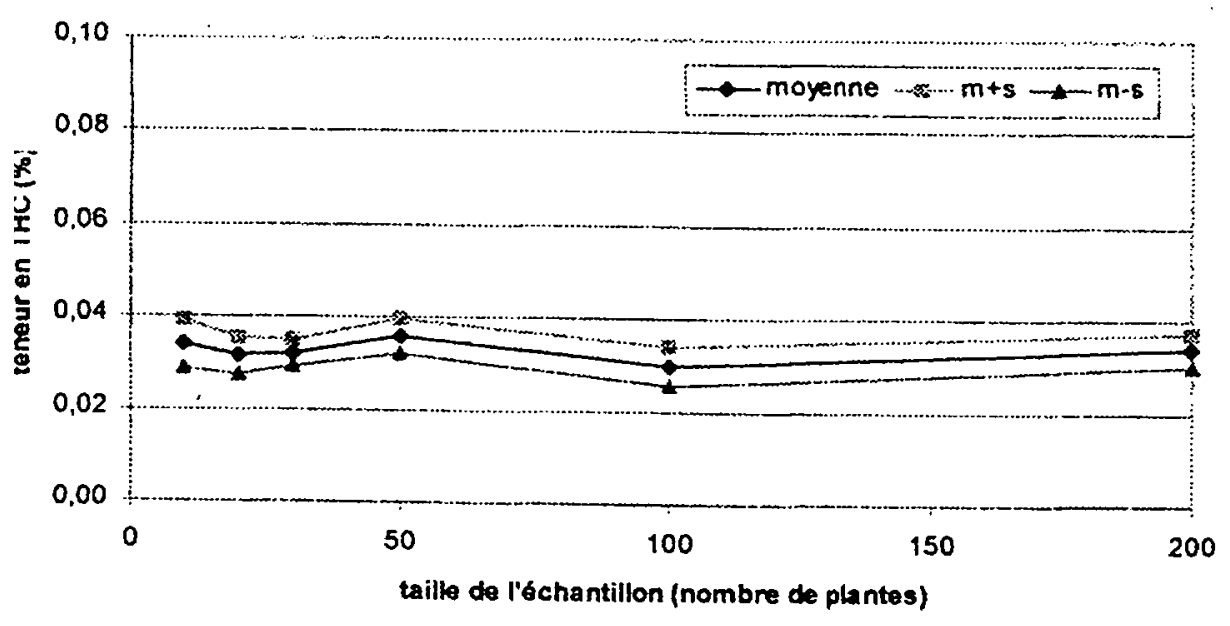

Figure 2 : Fluctuation de la teneur en $\Delta-9-T H C$ en fonction de la taille de l'échantillon. Variété Fédora 17 Prélèvement fin floraison des tiers supérieurs - Année 2000.
Le nombre de plantes dans un échantillon est donc un élément primordial à l'expression d'un résultat fiable de la teneur en $\triangle-9$-THC d'une population.

D'une manière générale, l'influence du nombre de plantes augmente avec l'accroissement de la teneur en $\Delta$-9-THC de la variété ; cette influence devient quasi nulle pour les variétés à faible, voire très faible, teneur en $\Delta-9$-THC. Dans ce dernier cas, et seulement dans celui-là, le nombre de plantes dans l'échantillon peut alors être réduit.

Un minimum de 100 plantes assure une grande sécurité pour le résultat, pour la plupart des variétés ;

50 plantes n'assurent une sécurité relative que pour des variétés dont la teneur en $\Delta-9$-THC est faible et homogène.

Dans la nouvelle méthode communautaire pour la détermination quantitative du $\Delta-9$-THC (3), on retrouve pour la procédure $A$ : 50 plantes et pour la procédure $B: 200$ plantes. Les résultats des expériences rapportées ci-dessus montrent que ces deux protocoles n'aboutissent à un même résultat que dans le cas de variétés sélectionnées présentant une teneur particulièrement faible en $\triangle-9-T H C$ et pour lesquelles un contrôle pourrait, d'ailleurs, être considéré comme superflu. Dans tous les autres cas, il est évident que les deux procédures ne peuvent jamais conduire au même résultat.

\section{Influence de la partie prélevée :}

Il est classiquement admis que les feuilles de chanvre contiennent moins de $\Delta-9$-THC que les inflorescences. Il est donc logique de penser que tout prélèvement conduisant à une distorsion (de poids) entre les feuilles et les inflorescences par rapport à une méthode classique (la méthode du 1/3 supérieur donne à peu près toujours les mêmes rapports) entraînera une modification de la teneur en $\Delta-9-T H C$ de l'échantillon.

Plusieurs essais réalisés au cours des dernières années ont porté d'une part sur la séparation des feuilles et des inflorescences et, d'autre part, sur l'influence que peuvent avoir certaines pratiques culturales sur la teneur en $\triangle$-9-THC.

\section{a) Comparaison de la teneur en $\triangle-9$-THC dans les} feuilles, les inflorescences et le tiers supérieur :

Au cours des années 1998 à 2000, les tiers supérieurs de plantes de différentes variétés ont été récoltés. Sur une partie aliquote, séparation des feuilles et des inflorescences; pesée individuelle des deux fractions. Trois lots de matières premières sont donc analysés : tiers supérieur complet (constitué des feuilles et des inflorescences), feuilles seules du tiers supérieur et inflorescences seules du tiers supérieur. Chaque lot est pesé et 
la teneur en $\Delta-9$-THC y est déterminée. Les résultats obtenus sont colligés dans les colonnes de gauche du Tableau I. Disposant à la fois du poids des inflorescences et de celui des feuilles d'une part et, d'autre part de la teneur en $\triangle-9$-THC de chacune de ces deux parties, il est possible de calculer la teneur théorique du tiers supérieur ainsi reconstitué. Les résultats sont rapporté dans la colonne de droite du Tableau I.

Le Tableau I fait bien ressortir que la teneur en $\Delta-9$ THC est :

- 2 à 4 fois plus élevée dans les inflorescences que dans les feuilles ;

- 2 fois plus élevée dans les inflorescences que dans les tiers supérieurs ;

- identique pour les valeurs mesurées ou calculées dans les tiers supérieurs.

b) Influence que peuvent avoir certaines pratiques culturales sur la teneur en $\triangle-9-$ THC :

A l'opposé l'une de l'autre, deux pratiques culturales donnent des plantes dont les développements sont très différents :

- une culture semée à $50 . \mathrm{Kg} / \mathrm{Ha}$ (densité industrielle) donne des plantes non ramifiées dont les parties vertes sont assez réduites ; ces cultures sont mises en place pour la production industrielle des fibres de la tige ;

- une culture semée à $1,5 \mathrm{Kg} / \mathrm{Ha}$ (faible densité) donne des plantes très ramifiées dont les inflorescences et la masse foliaire sont développées ; ces cultures sont d'ailleurs mises en place, entre autre, pour la production industrielle de semences.

Le Tableau II ci-dessous présente les résultats expérimentaux comparant la teneur en $\Delta-9-\mathrm{THC}$ dans la plante entière, au niveau du $1 / 3$ supérieur, et dans les parties hautes de la plante (chaque résultat correspond à la moyenne de 20 plantes, identiques dans chaque série d'essai).
Les plantes issues des cultures établies selon une densité importante présentent une teneur en $\Delta-9$-THC comparable au niveau de la plante entière et du tiers supérieur, et un peu plus élevée pour la partie « $« 0 \mathrm{~cm} »$. Ces résultats sont normaux car les parties vertes (feuilles et inflorescences) coïncident avec le tiers supérieur dont les $« 30 \mathrm{~cm} »$ représentent la majeure partie.

Pour les plantes issues de cultures mises en place selon une faible densité, les ramifications, le développement foliaire et celui du tiers supérieur sont importants. Il semble exister un certain gradient positif (de la plante entière à la sommité) dans les teneurs en $\Delta-9-T H C$ de ces différentes parties.

Ces différents résultats montrent que, pour une même variété, la teneur en $\Delta-9-$ THC varie non seulement en fonction de la partie prélevée, mais aussi en fonction du développement de la plante.

Dans tous les cas, il semble que la mesure du $\triangle-9-$ THC au niveau du $1 / 3$ supérieur indique la teneur réelle de la plante entière et donc de la culture, alors que la mesure au niveau des «30 $\mathrm{cm} »$ supérieurs conduit à déterminer la teneur maximale dans la plante en ce constituant, ce qui n'est pas la même chose.

Dans la nouvelle méthode communautaire pour la détermination quantitative du $\Delta-9-\mathrm{THC}(3)$, est noté pour la procédure $\mathrm{A}$ : prélèvement d' «une partie de $30 \mathrm{~cm}$ contenant au moins une inflorescence femelle pour chaque plante sélectionnée» et pour la procédure $B$ : «prélèvement du tiers supérieur de chaque plante sélectionnée». De même que

Tableau II : Comparaison de la teneur en $\Delta-9-T H C$ à différents niveaux de la plante en fonction de son développement. Moyenne de 20 analyses.

\begin{tabular}{|c|c|c|}
\hline & Faible densité & Densité industrielle \\
\hline Plante entière & 0,162 & 0,067 \\
\hline $\mathbf{1 / 3}$ supérieur & 0,171 & 0,068 \\
\hline $\mathbf{3 0} \mathbf{~ c m}$ & 0,214 & 0,075 \\
\hline
\end{tabular}

Tableau I : Comparaison de la teneur en $\Delta-9-T H C$ dans différentes parties de plusieurs variétés de Chanvre. Moyenne de 20 analyses.

\begin{tabular}{|c|c|c|c|c|c|}
\hline \multicolumn{2}{|c|}{} & \multicolumn{3}{|c|}{ Teneur (\%) en THC } \\
\hline Année & Variété & feuilles & $\begin{array}{c}\text { mesurée dans les } \\
\text { inflorescences }\end{array}$ & tiers supérieurs & $\begin{array}{c}\text { calculée dans le } \\
\text { tiers supérieur }\end{array}$ \\
\hline $\mathbf{1 9 9 8}$ & Futura 75 & 0,034 & 0,138 & 0,075 & 0,070 \\
\hline & Futura & 0,152 & 0,589 & 0,295 & 0,298 \\
\hline $\mathbf{1 9 9 9}$ & Fédora 17 & 0,032 & 0,099 & 0,051 & 0,049 \\
\hline & Fédora 19 & 0,108 & 0,212 & 0,139 & 0,142 \\
\hline $\mathbf{2 0 0 0}$ & Futura 75 & 0,058 & 0,089 & 0,067 & 0,065 \\
\hline & Futura & 0,051 & 0,180 & 0,089 & 0,088 \\
\hline
\end{tabular}


pour le nombre de plantes à prélever, les résultats des expériences rapportées ci-dessus montrent que ces deux protocoles ne peuvent conduire qu'à des résultats différents.

\section{Influence de la date de prélèvement :}

Cet essai est réalisé depuis plusieurs années et concerne plusieurs variétés ayant des teneurs moyennes en $\Delta-9$-THC assez différentes.

La méthode d'échantillonnage retenue correspond à la méthode $B$ ( $1 / 3$ supérieur ; 200 plantes).

Les résultats (année 2000) sont présentés sur la Figure 3.

D'une manière générale, il ressort que la teneur en $\Delta$-9-THC :

- croît en début de cycle ;

- observe un pic en début de floraison ;

- décroît pendant la période de la floraison ;

- marque un nouveau pic en fin de floraison ;

- devient difficile à interpréter après la fin de floraison (parfois stagne, parfois monte, parfois décroît, mais ce résultat n'est visible qu'en comparaison pluri-annuelle) ;

- est stable et constamment nulle dans le cas des variétés sélectionnées pour leur absence de $\Delta-9$-THC.

En se référant à ce qui a été précédemment exposé, on peut observer une certaine logique dans ces résultats. En effet, on a, en début de cycle, uniquement des feuilles, donc la teneur en $\Delta-9-$ THC est faible.

Dès que la floraison débute, la mesure est effectuée sur un mélange de feuilles et d'inflorescences, il est donc normal d'avoir un accroissement important.

Ce qui se passe durant la floraison est plus difficile à interpréter, mais correspond à ce que l'on observe d'une manière générale chez le chanvre durant cette période, à savoir des modifications importantes de la physiologie de la plante, et plus particulièrement un ralentissement important des métabolismes.

L'accroissement en fin de floraison est également logique ; il correspond à la perte des feuilles (qui tombent au sol), augmentant ainsi la proportion de l'inflorescence.

La fin de cycle confirme le point précédent. En effet, cette période coïncide avec la chute de feuilles pendant la maturation des graines (la part de l'inflorescence augmente donc encore). Néanmoins, en cas de conditions climatiques difficiles (pluie pour l'essentiel), on peut observer une reprise de végétation qui se traduit par une nouvelle pousse de feuilles. Dans d'autres cas (climat plutôt sec), la maturation des graines est bloquée, le métabolisme est bloqué, et la teneur en THC décroît.

Les deux Figures suivantes présentent les évolutions, d'une part, de la teneur en $\triangle-9-\mathrm{THC}$ (en \%) au cours du cycle végétatif (Figure 4) et, d'autre part, de la quantité globale en $\triangle$-9-THC de l'échantillon (Figure 5).

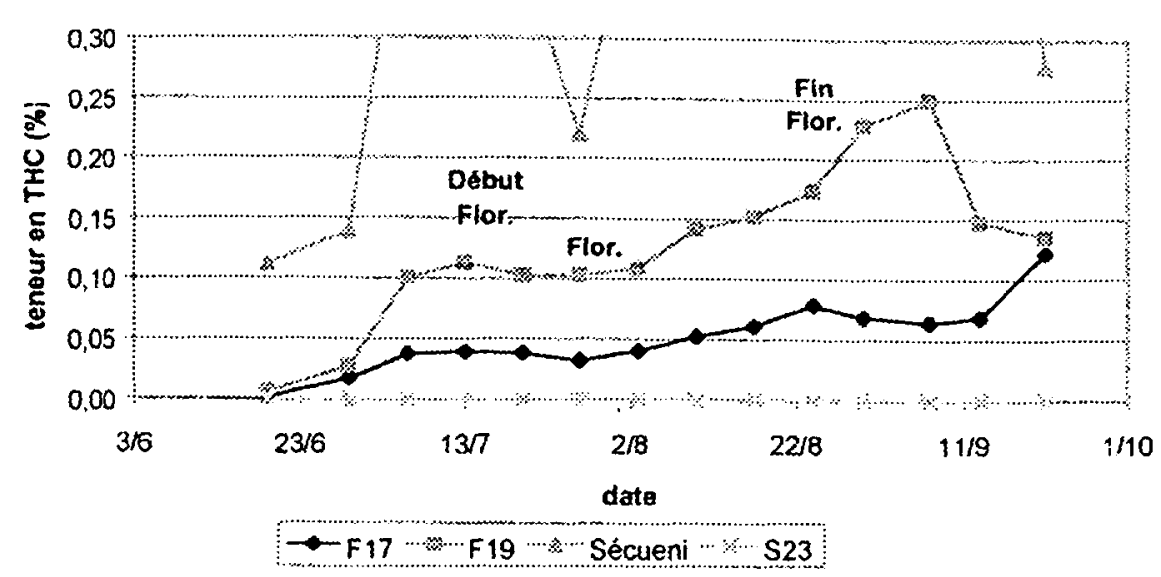

Figure 3 : Variations de la teneur en $\triangle-9-T H C$ en fonction du cycle végétatif de la plante. Comparaison du comportement de différentes variétés : Fédora 17 et 19, Sécuéni (variété roumaine non inscrite sur la liste des variétés de chanvre destinées à la production de fibres), Santhica 23.

On constate que l'évolution du pourcentage de $\Delta-9$-THC de l'échantillon au cours du cycle est conforme à ce qui a été décrit précédemment. Mais on constate aussi que la quantité globale de $\Delta-9-$ THC (produit du $\%$ de $\Delta-9$-THC par le poids de l'échantillon) évolue différemment. La quantité maximale se situe en début de floraison. Ce stade se déroule sur une période courte (quelques jours) et est donc difficile à exploiter. Il convient de noter un deuxième pic lors de la fin de la floraison.

$\mathrm{Si}$ on souhaite déterminer cette valeur maximale, il faut donc se placer en début de floraison, avec toutes les incertitudes (stade très fugace) liées aux variations des résultats autour de ce moment précis. Il est aussi possible de réaliser les prélèvements à la fin de la floraison, les variations sont alors très limitées et le résultat correspond à environ $2 / 3$ de ce qu'il est lors du début de la floraison.

Il est également intéressant de suivre le même type d'évolution de la teneur en $\Delta-9$-THC mais séparément dans les feuilles et dans les inflorescences. C'est ce que représente la Figure 6.

Les résultats de cette évolution comparée de la teneur en $\Delta$-9-THC dans les deux parties végétales confirment le point précédent, notamment :

- La teneur en $\Delta$-9-THC des feuilles croît, mais faiblement, on début de cycle ; cela correspond à la phase d'implantaion de la culture.

- La teneur en $\Delta-9$-THC des feuilles devient plus importante dès le début de la phase de croissance active et reste relativement stable pendant une phase assez longue (début de maturation des graines). A partir de ce stade, la teneur décroît, et cela peut être assimilé à la chute des feuilles (fin de la fonction de photosynthèse, transfert des assimilats (sucres, protéines) vers les graines pour nourrir celles-ci).

- De même, la teneur en $\Delta-9$-THC des inflorescences reste relativement stable durant la période de floraison.

Ces résultats permettent de confirmer que les proportions relatives de feuilles et d'inflorescences jouent pour beau- 


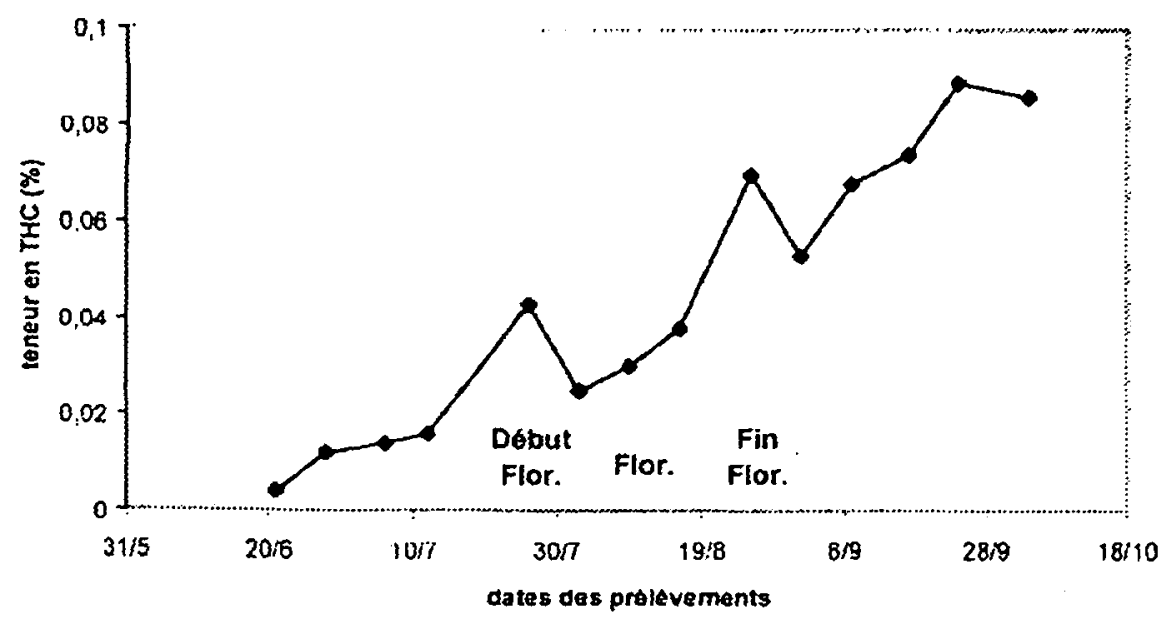

Figure 4 : Évolution de la teneur (\%) en $\Delta-9-T H C$ au cours du cycle végétatif. Variété Epsilon 68.

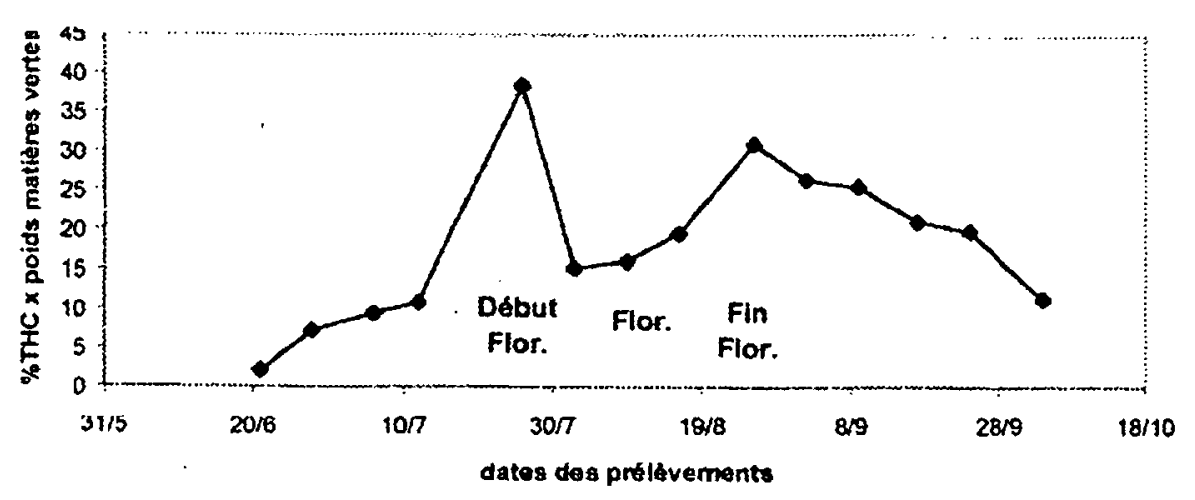

Figure 5 : Évolution de la quantité de $\Delta-9-7 H C$ au cours du cycle végétatif. Variété Epsilon 68.

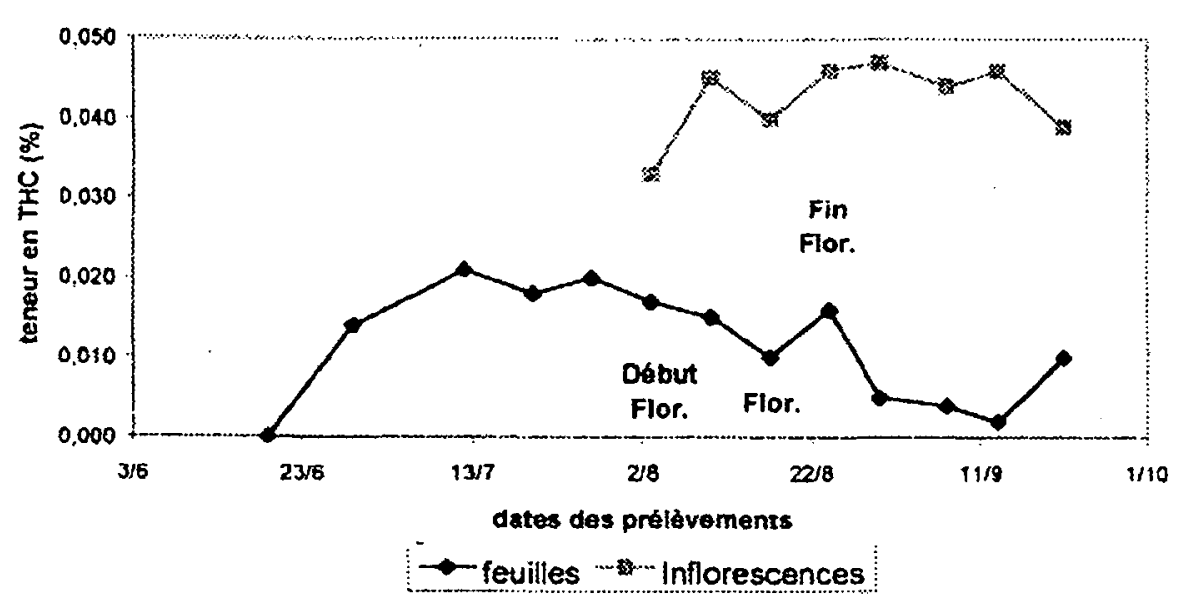

Figure 6 : Évolution de la teneur en $\Delta-9-T H C$ au cours du cycle végétatif, dans les feuilles et dans les inflorescences. Variété Fédora 17.

coup dans l'expression du résultat de la teneur en $\Delta-9-T H C$ au cours du cycle.

Dans la nouvelle méthode communautaire pour la détermination quantitative du $\Delta-9-T H C$ (3), on retrouve pour la procédure $\mathrm{A}$ : «le prélèvement s'effectue pendant la période comprise entre les vingt jours après le début et les dix jours après la fin de la floraison» et pour la procédure $B$ : «le prélèvement s'effectue au cours des dix jours suivant la fin de la floraison». Les résultats des expériences rapportées ci-dessus montrent que, du fait de la variabilité de la teneur en $\triangle-9-T H C$ au cours du cycle végétatif de la plante, ces deux protocoles ne peuvent conduire à un résultat reproductible que dans le cas où les prélèvements sont effectués au même stade, ce qui peut ne pas être toujours le cas étant donné la dualité proposée dans la méthode.

\section{Conclusion}

Les nombreuses analyses que nous avons réalisées depuis plus de deux décennies sur le chanvre cultivé licitement en France ont permis de mettre en évidence la grande variabilité de la teneur en $\Delta-9$-THC de cette plante. Cette variabilité est principalement due à des facteurs génétiques (existance de plusieurs types chimiques) mais, aussi, à des facteurs liés à la plante elle-même (selon son stade végétatif et selon les organes : absence de $\Delta-9-T H C$ dans les racines et les graines ; présence de $\Delta-9-\mathrm{THC}$, en proportions différentes, dans les feuilles et les sommités).

Depuis 1990, une méthode officielle de dosage était en vigueur. L'échantillonnage était réalisé par le prélèvement, à la fin de la floraison, des tiers supérieurs de 500 plantes. Seules étaient autorisées les cultures établies à partir de variétés dont la teneur en $\Delta-9$-THC n'était pas supérieure à $0,30 \%$.

En 2000, une nouvelle méthode officielle est entrée en vigueur. La teneur en $\triangle-9-T H C$ des variétés autorisées ne doit pas être supérieure à $0,20 \%$. Deux protocoles de prélèvement sont décrits ; l'un est utilisé pour contrôler la production; l'autre est mis en œuvre lors de la demande d'inscription d'une nouvelle variété. Ces deux protocoles diffèrent par le nombre de plantes prélevées, par la partie de la plante prélevée et par la période durant laquelle doivent se faire les prélèvements. Logiquement et expérimentalement, la méthode $\mathrm{A}$ ne peut conduire qu'à un résultat supérieur (et en tout cas plus variable et moins répétable) à celui obtenu avec la méthode B qui, lui, est proche de celui obtenu avec «l'ancienne» méthode.

Après les critiques doivent venir les propositions relatives à de nouvelles conditions d'échantillonnage applicables dans tous les cas. Ces nouvelles propositions feront l'objet d'un prochain travail.

Parallèlement aux recherches entreprises afin de proposer une amélioration des conditions d'échantillonnage, la sélection variétale se poursuit notamment en France. Elle permettra très prochainement la mise en culture exclusive de variétés dont la teneur en $\Delta-9$-THC reste très faible quelles que soient les conditions de culture.

\section{Références}

1. Fournier G. La sélection du chanvre à fibres (Cannabis sativa L.) en France. Chanvre et THC. C. R. Acad. Agric. Fr. $2000 ; 86: 209-217$.

2. Arrêté du 22 août 1990 portant application de l'article $\mathbf{R}$. 5181 du Code de la Santé Publique pour le cannabis. Journal Officiel de la République Française 4/10/1990.

3. Règlement (CE) $n^{\circ} 2860 / 2000$ de la Commission du 27 décembre 2000. Journal Officiel des Communautés européennes 28/12/2000. 